Running Head: CULTURE, MEMORY SHARING AND RELATIONSHIP CLOSENESS

Citation:

Guan, L., \& Wang, Q. (2020). Does sharing memories make us feel closer? The roles of memory type and culture. https://doi.org/10.31234/osf.io/7udy4

Does Sharing Memories Make Us Feel Closer? The Roles of Memory Type and Culture

\author{
Li Guan and Qi Wang \\ Cornell University
}

Correspondence may be addressed to Qi Wang, Department of Human Development, Cornell

University, MVR Hall, Ithaca, NY 14853-4401; telephone 607-255-9376; electronic mail qiwang@cornell.edu. 


\begin{abstract}
The present research used hypothetical scenarios to examine the effects of sharing different types of memories on perceived relationship closeness and how that is related to psychological well-being in a cross-cultural context. In two studies, European American and Asian participants (total $N=714$ ) reported their feelings of closeness to a conversation partner in hypothetical situations involving five types of information sharing: specific and general autobiographical memories, specific and general vicarious memories, and non-person information. Sharing memories led to greater feelings of closeness than sharing non-person information in both groups. Asians felt closer to the conversation partner than European Americans across all types of information sharing. Although sharing autobiographical and specific memories led to greater closeness than sharing vicarious and general memories in both groups, sharing specific autobiographical memories appeared most effective for relationship closeness for European Americans. Perceived relationship closeness following memory-sharing in hypothetical situations was positively associated with psychological well-being across cultures.
\end{abstract}

Keywords: autobiographical memory; vicarious memory; memory sharing; relationship closeness; culture 


\section{Does Sharing Memories Make Us Feel Closer? The Roles of Memory Type and Culture}

Sharing memories serves important social functions by facilitating relationship closeness between conversation partners. During the conversations, the partners reexperience past events together, exchange thoughts and feelings, express empathy towards each other, and form joint perspectives about what happened. These processes further contribute to the development and maintenance of relationship closeness (Bluck, 2003; Pillemer, 2001; Wang, 2013). Indeed, some researchers regard social bonding to be the primary function of human memory (Bruce, 1989; Nelson \& Fivush, 2004). Little is known about what factors influence the social functions of memory. In particular, does sharing different types of memory matter for how people feel about their relationship closeness following the conversation and if so, does culture play a role in moderating the process? Moreover, is the feeling of closeness from memory sharing indeed beneficial for individual well-being? To answer these questions, we set out to examine in a cross-cultural context how different types of memories autobiographical versus vicarious memories and specific versus general memories influence perceived relationship closeness following memory sharing in hypothetical situations and how the feeling of closeness is further linked to psychological well-being.

\section{Autobiographical versus Vicarious Memories}

Memories differ in the identity of their protagonists. Autobiographical memory has the rememberer him- or herself as the protagonist, involving the recollection of significant personal experiences from an individual's life (Brewer, 1986; Pillemer, 2001; Wang, 2013). In everyday life, autobiographical memories play an important role for developing and maintaining relationship closeness (Alea \& Bluck, 2007; Bluck, Alea, Habermas, \& Rubin, 2005; Wang, 2004). Indeed, 44\% - 75\% of everyday conversations involve sharing personal experiences, at least in the Western cultural context (Beike, Brandan, \& Cole, 2016; Pasupathi \& Cartensen, 2003). Such memory conversations often involve not just families 
and friends but also strangers (Demiray, Mischler, \& Martin, 2019). When a conversation draws information from one's own experiences, it often appears credible and convincing and can thus engage listeners and induce their empathic responses, especially from listeners who have had similar experiences (Pillemer, 2001). Furthermore, sharing autobiographical memories provides the listeners with self-revealing information, which serves as a natural context for exchanging thoughts, emotions, and opinions with others (Alea \& Bluck, 2003; Cohen, 1998; Demiray et al., 2019; Rasmussen \& Habermas, 2011). These processes facilitate relationship development and intimacy.

In contrast to autobiographical memories, vicarious memories have others rather than the self as the protagonist, involving the recollection of other people's experiences, such as events that happened to family members, friends, and even celebrities and the society at large in the form of news events (Pillemer, Steiner, Kuwabara, Thomsen, \& Svob, 2015; Reese, Fivush, Merrill, Wang, \& McAnally, 2017; Wang, 2018). Although vicarious memories share similar characteristics with autobiographical memories in content focus and emotional reactions (Pillemer, et al., 2015; Wang, 2006), they are lower in phenomenological experiences such as emotional intensity, vividness, clarity, and positivity than autobiographical memories (Thomsen \& Pillemer, 2017).

Like autobiographical memories, vicarious memories can create and strengthen social bonds among individuals (Miller, 2009; Wang, 2013). Still, given the physical absence of the protagonist from the context of the conversation and the low degree of self-disclosure by the memory sharer, sharing vicarious memories may not be as influential as sharing autobiographical memories on interpersonal connection between the conversation partners. Indeed, vicarious memories are viewed as less important than autobiographical memories in affecting relationships (Pillemer et al., 2015). Whether autobiographical and vicarious 
memories variably influence perceived relationship closeness in the context of memory sharing remains an empirical question.

\section{Specific versus General Memories}

Memories further differ in specificity. No matter whether the protagonist is oneself or another person, memories can be of one-moment-in-time events that occurred at a particular place and time in the past typically lasting no longer than a day - specific memories, or repeated events occurring regularly during a period of time - general memories (Conway \& Pleydell-Pearce, 2000; Pillemer, 2001; Rubin, 2005; Tulving, 2002; Williams \& Broadbent, 1986). ${ }^{1}$ Specific memories involve the recollection of unique and discrete episodes, often accompanied by a sense of mentally re-experiencing the past. In contrast, general memories often involve the provision of semantic or scripted information without evoking details from specific instances.

Researchers suggest that compared with general memories, specific memories contain more vivid sensory and emotional details and therefore are more likely to captivate and engage listeners (Alea \& Bluck, 2003; Baron \& Bluck, 2009). Sharing specific memories also allows individuals to effectively convey personal meanings of particular past events (Pillemer, 2001). Furthermore, although both specific and general memories can provide listeners with information about the memory protagonist (e.g., preferences, occupation, relationships), sharing specific memories can evoke a sense of mental time travel for the conversation partners to re-experience a past moment together (Beike, Cole, \& Merrick, 2017; Rubin, 2005; Wang, 2013). Thus, specific memories may better facilitate relationship closeness than general memories in the context of memory sharing.

\footnotetext{
${ }^{1}$ There are also events that are unique one-time events and yet lasted for a period of time (e.g., a family trip to Paris). Such events often encompass many single-day specific events (e.g., visiting the Eiffel Tower on a particular day). We did not include memories for this type of events in the current study.
} 
To test this question, Beike et al. (2016) asked unacquainted pairs of participants to share specific autobiographical memories, general self-information (e.g., a summer job), or nonself-related information (e.g., answering trivia questions). Participants then reported their feelings of closeness with their conversation partners. Although sharing specific autobiographical memories facilitated greater relationship closeness than sharing nonselfrelated information, it did not differ from sharing general self-related information. Yet, in another study, Beike et al. (2017) found that sharing specific, but not general, memories of co-experienced events facilitated intimacy in close relationships. In contrast, Waters, Bauer and Fivush (2014) found that personal narratives of recurring events (i.e., general memories) conveyed a greater sense of relationship closeness than narratives of single events (i.e., specific memories), although it is unclear whether this finding applies to the conversational context given the influence of contextual factors on memory functions (Kulkofsky, Wang, \& Hou, 2010). Obviously, additional research is required to examine the effect of memory specificity on relationship closeness following memory sharing.

\section{Culture, Memory Sharing, and Relationship Closeness}

Culture influences how people use memories in everyday life and for relationship purposes in particular (for a review, see Alea \& Wang, 2015). In Western cultures, relationships tend to be voluntary and individuals have much freedom to choose their friends and partners based on their personal preferences (Yuki \& Schug, 2012; Yuki et al., 2007). Given the high relational mobility, actively developing and maintaining relationships is required in this cultural context, and sharing memories - particularly memories for unique personal events - is considered an effective means to achieve the purpose (Chen, 1995; Kito, 2005; Wang, 2013). In comparison, in many Asian cultures, relationships are largely defined by geographical vicinity or circumstances of birth and therefore tend to be more stable and require less maintenance through means such as memory sharing (Brint, 2001; Kito, Yuki \& 
Thomson, 2017; Wang, 2004). Indeed, European American individuals report sharing memories - specific autobiographical memories in particular - more frequently than do Asians for social bonding purposes (Kulkofsky et al., 2010; Kulkofsky, Wang, \& Koh, 2009; Maki, Kawasaki, Demiray, \& Janssen, 2015; Schug, Yuki, \& Maddux, 2010).

Culture further influences individuals' sense of self in relation to others, which may, in turn, affect feelings of closeness following memory sharing. Whereas Western cultures greatly value independence and personal uniqueness, many Asian cultures prioritize interdependence and social harmony (Markus \& Kitayama, 1991; Wang, 2013). Accordingly, when recalling personal experiences, Asians often focus on social interactions and group activities, whereas European Americans often focus on their own roles and perspectives (Wang, 2001, 2006; Wang \& Conway, 2004; Wang \& Ross, 2007). When interacting with others, Asians are more sensitive to social cues and focus more on consensus building than European Americans (Setlock, Fussell, \& Neuwirth, 2004; Wang H-C, Fussell, \& Setlock, 2009). Furthermore, individuals who are more interdependently oriented perceive their relationships more optimistically than those who are more independently oriented (Cross \& Morris, 2003). It is thus possible that social exchanges such as memory sharing can elicit greater feelings of closeness among Asians than European Americans.

\section{Perceived Relationship Closeness and Psychological Well-Being}

Relationship quality is essential for psychological well-being (Baumeister \& Leary, 1995; Umberson, Crosnoe, \& Reczek, 2010). Whereas supportive relationships are associated with psychosocial adjustments, unsupportive and neglectful relationships lead to disruptions to well-being (e.g., Repetti, Taylor, \& Seeman, 2002; Townsend, McCracken, \& Wilton, 1988; Umberson et al., 2010). More generally, regardless of culture, social connectedness is a basic human need that must be fulfilled for individuals to maintain psychological functioning (Ryan \& Deci, 2000). 
Importantly, individuals' interpretations of social interactions and the associated relationship qualities - actual or hypothetical - have implications for their psychosocial adjustments (Song, Koh \& Wang, 2018; Warren, Emde, \& Sroufe, 2000). For example, children who perceive hypothetical interpersonal interactions more in terms of caring themes of affiliation, reparation, and prosocial behaviors exhibit more positive long-term trajectories in development, compared with children who perceive more negative themes such as aggression and destruction (Warren et al., 2000; Zahn-Waxler, Park, Usher, Belouad, Cole \& Gruber, 2008). In the context of memory sharing, individuals who are better able to derive positive meanings from the conversation and perceive greater closeness with the conversation partner may benefit more from the social exchange.

\section{The Present Research}

The present research examined in a cross-cultural context the effect of memory protagonist and specificity during memory sharing on developing relationship closeness and the relation of perceived closeness to psychological well-being. In two studies, European American and Asian participants recruited through Amazon Mechanical Turk reported their feelings of closeness to a conversation partner in hypothetical scenarios that involved five types of information sharing: specific and general autobiographical memories, specific and general vicarious memories, and non-person information that did not involve any protagonist (e.g., talking about the weather).

Notably, memory sharing serves both the functions of nurturing existing relationships and forming new relationships (Bluck et al., 2005; Rasmussen \& Habermas, 2011). We focused on new relationships here to eliminate the potential effect of pre-existing relationship qualities, which is especially important in a cross-cultural context (e.g., Sparks, Cunningham, \& Kritikos, 2016). Furthermore, the use of hypothetical scenarios allows the control of individual variations in memories to be shared and has been a useful approach to studying 
memory and other cognitive processes (Goyal, Adams, Cyr, Maass \& Miller, 2020; Wang \& Leichtman, 2000; Zhao \& Kushnir, 2019). Research has also shown that guided by their relationship schemas, individuals interpret hypothetical social interactions in line with their actual interpersonal experiences, which reveals their views of the self and others and predicts their psychological well-being (Oppenheim, 2006; Song et al., 2018; Wang \& Ross, 2005; Zahn-Waxler et al., 2008). We collected data online to obtain samples from demographically diverse populations.

Given the social functions of memory sharing (Nelson \& Fivush, 2004; Pillemer, 2001; Wang, 2013), we expected that in both cultural groups, sharing memories in hypothetical situations would elicit greater feelings of closeness than sharing non-person information. On the other hand, given their interdependent value orientation and their attentiveness to social cues during interpersonal exchanges (Markus \& Kitayama, 1991; Setlock et al., 2004; Wang H-C et al, 2009), Asians would perceive greater relationship closeness in all conversational contexts than European Americans. Furthermore, in line with the literature (Alea \& Bluck, 2003, Pillemer et al., 2015), we expected that sharing autobiographical and specific memories would lead to greater perceived relationship closeness than sharing vicarious and general memories in both cultural groups, and that this would be particularly true for European Americans given the special value of specific autobiographical memories for social bonding in Western cultures (Kulkofsky et al., 2010; Maki et al., 2015; Wang, 2004).

We first tested these hypotheses in Study 1 and then replicated the findings in Study 2. In addition, we measured in Study 1 cultural value orientations at the individual level and expected that those who exhibited a greater interdependent value orientation would perceive greater relationship closeness following imagined memory sharing. In turn, greater relationship closeness would be related to better psychological well-being across cultures. 


\section{Study 1}

\section{Method}

Participants. A total of 410 participants were recruited through Amazon Mechanical Turk, including 272 self-identified as White or European American (hereinafter referred to as European Americans; 134 males and 138 females; all except 15 were located in the US) and 138 self-identified as Asian or Asian American (hereinafter referred to as Asians; 102 males and 36 females; 74\% were located outside the US). Participants' ages ranged from 19 to 68 years $(M=34.66, S D=10.79)$. Among the participants, 74 received high school education or less, 174 had college degrees, and 162 had graduate degrees. A power analysis (G*Power; Faul, Erdfelder, Lang \& Buchner, 2007) showed that a sample size of 266 would be needed to achieve a power of 0.9 to detect effects with a size of $f=0.10$ and $\alpha=0.05$. We maximized our sample size within funding constraints. An additional 42 participants did not complete the survey or follow the instructions and another 95 participants self-identified as belonging to other ethnic groups; those participants were excluded from the study.

Procedure and measures. Participants took part in a 30-min online survey. They were asked to imagine that they newly met a person who shared information with them in a short conversation. They were then presented in random order with 10 conversational scenarios that included five types of information sharing (see Table 1). The length of the scenarios was similar across the different types of information sharing; it ranged from 51 to 59 words $(M=55.3, S D=2.4)$.

Table 1

Conversation Scenario Excerpts by Information Sharing Type

\begin{tabular}{lll}
$\begin{array}{l}\text { Information } \\
\text { sharing type }\end{array}$ & Definition & Scenario excerpts \\
\hline Specific & Memory for one's own & "My 21 2 st birthday was so much fun... I
\end{tabular}




\begin{tabular}{|c|c|c|}
\hline $\begin{array}{l}\text { autobiographical } \\
\text { memory }\end{array}$ & $\begin{array}{l}\text { experience occurring at a } \\
\text { particular place and time }\end{array}$ & $\begin{array}{l}\text { had a blast..." } \\
\text { "I saw my favorite artist... I kept on } \\
\text { jumping up and down..." }\end{array}$ \\
\hline $\begin{array}{l}\text { General } \\
\text { autobiographical } \\
\text { memory }\end{array}$ & $\begin{array}{l}\text { Memory for one's own } \\
\text { experience occurring } \\
\text { repeatedly during a period } \\
\text { of time }\end{array}$ & $\begin{array}{l}\text { "I love to be outdoors... In the summer } \\
\text { and spring I enjoy kayaking..." } \\
\text { "I work at Bank of Ozarks... It's very } \\
\text { laid back..." }\end{array}$ \\
\hline $\begin{array}{l}\text { Specific } \\
\text { vicarious } \\
\text { memory }\end{array}$ & $\begin{array}{l}\text { Memory for someone else's } \\
\text { experience occurring at a } \\
\text { particular place and time }\end{array}$ & $\begin{array}{l}\text { "My roommate went to my hometown... } \\
\text { she felt welcomed..." } \\
\text { "My niece ... went to the park and then } \\
\text { to the swimming area..." }\end{array}$ \\
\hline $\begin{array}{l}\text { General } \\
\text { vicarious } \\
\text { memory }\end{array}$ & $\begin{array}{l}\text { Memory for someone else's } \\
\text { experience occurring } \\
\text { repeatedly during a period } \\
\text { of time }\end{array}$ & $\begin{array}{l}\text { "I have five nieces and nephews... before } \\
\text { you know it, they've grown up." } \\
\text { "Ada is one of my best friends... She has } \\
\text { studied German since she was six years } \\
\text { old..." }\end{array}$ \\
\hline $\begin{array}{l}\text { Non-person } \\
\text { information }\end{array}$ & $\begin{array}{l}\text { Information not involving } \\
\text { any protagonist }\end{array}$ & $\begin{array}{l}\text { "New York City has a humid subtropical } \\
\text { climate..." } \\
\text { "The iPhone } 8 \text { is released on September } \\
22^{\text {nd }}, 2017 \ldots \text {..." }\end{array}$ \\
\hline
\end{tabular}

Following each conversation description, participants were asked to rate in response to four questions their feelings of closeness to the conversation partner. The first question was adapted from the Inclusion of Other in Self Scale (IOS; Aron, Aron, \& Smollan, 1992), which is a one-item pictorial measure for people's sense of interpersonal interconnectedness. Participants chose one of the seven Venn-like diagrams, each showing different degrees of overlap between two circles labelled "Self" and "Other." The IOS has good test-retest reliability and predictive validity and has proved to be a valid measure of closeness with intimate partners as well as strangers (Aron et al., 1992). 
Following rating on the IOS Scale, participants responded to the question "How likely would you be to use the term "we" to characterize you and your conversation partner?" by rating on a 7-point scale from 1 (not at all) to 7 (extremely) (Cialdini, Brown, Lewis, Luce, \& Neuberg, 1997). They then responded to two additional questions adapted from the Subjective Closeness Index (Gächter, Starmer, \& Tufano, 2015), including "Relative to all your other relationships (both same and opposite sex), how would you characterize your relationship with your conversation partner?" and "Relative to what you know about other people's close relationships, how would you characterize your relationship with your conversation partner?" Participants rated on 7-point scales from 1 (not at all close) to 7 (extremely close).

Cronbach's alphas for the ratings on the IOS Scale and the three subsequent questions used to assess the participants' perceived closeness with the conversation partner ranged from .94 to .96 across the 10 conversations. The four ratings were thus averaged for each conversation to index the degree of perceived closeness following the conversation.

In addition, participants completed the Self-Construal Scale and the Flourishing Scale and provided demographic information. The Self-Construal Scale (SCS; Singelis, 1994) consists of two subscales that measure individuals' value orientation towards independence (e.g., "I enjoy being unique and different from others in many respects") and interdependence (e.g., "I often have the feeling that my relationships with others are more important than my own accomplishments"), respectively. Each subscale includes 15 items, and participants rated each item from 1 (strongly disagree) to 7 (strongly agree). The SCS has been used widely in cross-cultural research (e.g., Neff, Pisitsungkagarn, \& Hsieh, 2008; Wang, 2001; Yoon, Hasher, Feinberg, Rahhal, \& Winocur, 2000;). Cronbach's alpha in the current sample was .83 for independence and .87 for interdependence. A composite score of independent score minus interdependent score was often used for analysis (e.g., Holland, Roeder, Rick, 
Brandt, \& Hannover, 2004). Given our focus on relationship closeness, we computed a composite score of interdependent score minus independent score to index the degree of an interdependent value orientation and submitted the composite score to relevant analyses.

The Flourishing Scale (Diener et al., 2009) tests individuals' psychological resources and strengths. It consists of 8 items to assess various aspects of human functioning, such as relationships, self-esteem, purpose, and optimism (e.g., "I am competent and capable in the activities that are important to me"). Participants responded to each item on a 7-point scale (1=strongly disagree; 7 = strongly agree). Cronbach's alpha for the scale in the current sample was .92 . The total score across the 8 items (maximum $=56$ ) was used to index the participants' psychological well-being.

\section{Results}

Preliminary analyses showed that European Americans $\left(M_{\text {age }}=37.16, S D=11.62\right)$ were on average older than Asians $\left(M_{\text {age }}=29.66, S D=9.15\right), t(338)=7.14, p<.001, d=.72$, and there was a greater percentage of females (51\%) in the European American sample than in the Asian sample $(19 \%), \chi^{2}(1, N=410)=22.77, p<.001, \varphi=.24$. Asians had higher educational levels than did European Americans, $\chi^{2}(2, N=410)=118.45, \mathrm{p}<.001, \varphi=.54$. All subsequent analyses were conducted both with and without the demographic variables as covariates, which yielded identical patterns of results. We report the results of analyses with the covariates.

\section{Memory characteristics and perceived closeness across cultures. In this} section, we examined the participants' perceived closeness with the imagined conversation partner as a function of the type of information sharing and culture. The closeness ratings were averaged between the two scenarios for each type of information sharing and submitted to analyses. The means and standard deviations are presented in Table 2 by culture and information type. 
Table 2

Means and Standard Deviations of Perceived Closeness

\begin{tabular}{|c|c|c|c|c|c|c|c|}
\hline & & \multicolumn{2}{|c|}{ All } & \multicolumn{2}{|c|}{$\begin{array}{l}\text { European } \\
\text { Americans }\end{array}$} & \multicolumn{2}{|c|}{ Asians } \\
\hline & & Mean & SD & Mean & $\mathrm{SD}$ & Mean & SD \\
\hline \multirow[t]{2}{*}{ Specific } & Autobiographical & 3.83 & 1.56 & 3.34 & 1.50 & 4.78 & 1.21 \\
\hline & Vicarious & 3.55 & 1.60 & 2.97 & 1.44 & 4.68 & 1.24 \\
\hline \multirow[t]{2}{*}{ General } & Autobiographical & 3.62 & 1.53 & 3.06 & 1.38 & 4.72 & 1.18 \\
\hline & Vicarious & 3.63 & 1.51 & 3.13 & 1.40 & 4.63 & 1.20 \\
\hline Control & Non-person & 2.98 & 1.72 & 2.38 & 1.42 & 4.16 & 1.65 \\
\hline
\end{tabular}

First, to test whether sharing memories would result in feeling closer to the conversation partner than sharing non-person information, a 2(culture: European American versus Asian) x 2(conversational content: memory versus non-person information) repeatedmeasures ANOVA was conducted on the closeness rating, with culture as a between-subjects factor, conversational content as a within-subjects factor, and an error term accounting for within-individual variance. Age, gender, and education were included in the model. There were main effects of culture, $F(1,404)=51.07, p<.001, \eta_{\mathrm{p}}^{2}=.112$, and conversational content, $F(1,408)=163.20, p<.001, \eta_{\mathrm{p}}{ }^{2}=.286$, and a marginally significant Culture $\mathrm{x}$ Conversational content interaction, $F(1,408)=3.09, p=.079, \eta_{\mathrm{p}}^{2}=.008$. Asians perceived greater closeness than did European Americans regardless of the type of information sharing. Both European Americans and Asians felt closer to the imagined conversation partner when sharing memories than when sharing non-person information, with the difference being larger for European Americans, $F(1,271)=126.90, p<.001, \eta_{\mathrm{p}}^{2}=.319$, than for Asians, $F(1,137)$ $=37.88, p<.001, \eta_{\mathrm{p}}^{2}=.217$. 
Then, to examine the influence of sharing different types of memory on perceived relationship closeness, we focused on the four types of memory sharing in subsequent analyses. We conducted a 2(culture: European American vs. Asian) x 2(specificity: specific vs. general) x 2(protagonist: autobiographical vs. vicarious) repeated-measures ANOVA on the closeness rating, with culture as a between-subjects factor, memory specificity and memory protagonist as within-subjects factors, and an error term accounting for withinindividual variance. Age, gender, and education were included in the model. There were main effects of culture, $F(1,404)=48.99, p<.001, \eta_{\mathrm{p}}{ }^{2}=.108$, memory protagonist, $F(1,408)=$ $15.81, p<.001, \eta_{\mathrm{p}}^{2}=.037$, and memory specificity, $F(1,408)=4.63, p=.032, \eta_{\mathrm{p}}^{2}=.011$, qualified by a two-way interaction between specificity and protagonist, $F(1,408)=23.30, p$ $<.001, \eta_{\mathrm{p}}^{2}=.054$, and a three-way interaction between culture, specificity, and protagonist, $F(1,408)=11.18, p=.001, \eta_{\mathrm{p}}^{2}=.027$. The adjusted mean ratings from the model are shown in Figure 1.

Asians perceived greater closeness than did European Americans regardless of the type of memory sharing. Further repeated-measures ANOVAs were then conducted within each culture to examine the effects of memory protagonist and memory specificity on closeness ratings. In the Asian group, only the effect of protagonist was marginally significant, $F(1,137)=3.25, p=.074, \eta_{\mathrm{p}}{ }^{2}=.023$, whereby Asians felt closer to the imagined conversation partner when sharing autobiographical memories than when sharing vicarious memories. Memory specificity did not affect their perceived closeness. In the European American group, there was also a main effect of memory protagonist, $F(1,271)=12.74, p$ $<.001, \eta_{\mathrm{p}}^{2}=.045$, which was qualified by a Protagonist $\mathrm{x}$ Specificity interaction, $F(1,271)=$ 31.06, $p<.001, \eta_{\mathrm{p}}{ }^{2}=.103$. Pairwise comparisons showed that when sharing specific memories, European Americans felt closer to the partner when the conversation concerned autobiographical than vicarious events, $F(1,271)=35.12, p<.001, \eta_{\mathrm{p}}{ }^{2}=.115$, whereas there 
was no significant effect of memory protagonist when sharing general memories, $F(1,271)=$ $1.29, p=.26, \eta_{\mathrm{p}}^{2}=.005$. In addition, there was greater perceived closeness for sharing specific than general autobiographical memories, $F(1,271)=37.84, p<.001, \eta_{\mathrm{p}}^{2}=.123$, whereas there was greater perceived closeness for sharing general than specific vicarious memories, $F(1,271)=6.24, p=.013, \eta_{\mathrm{p}}^{2}=.023$. Overall, for European Americans, sharing specific autobiographical memories with an imagined partner led to greater perceived closeness than sharing other types of memories.

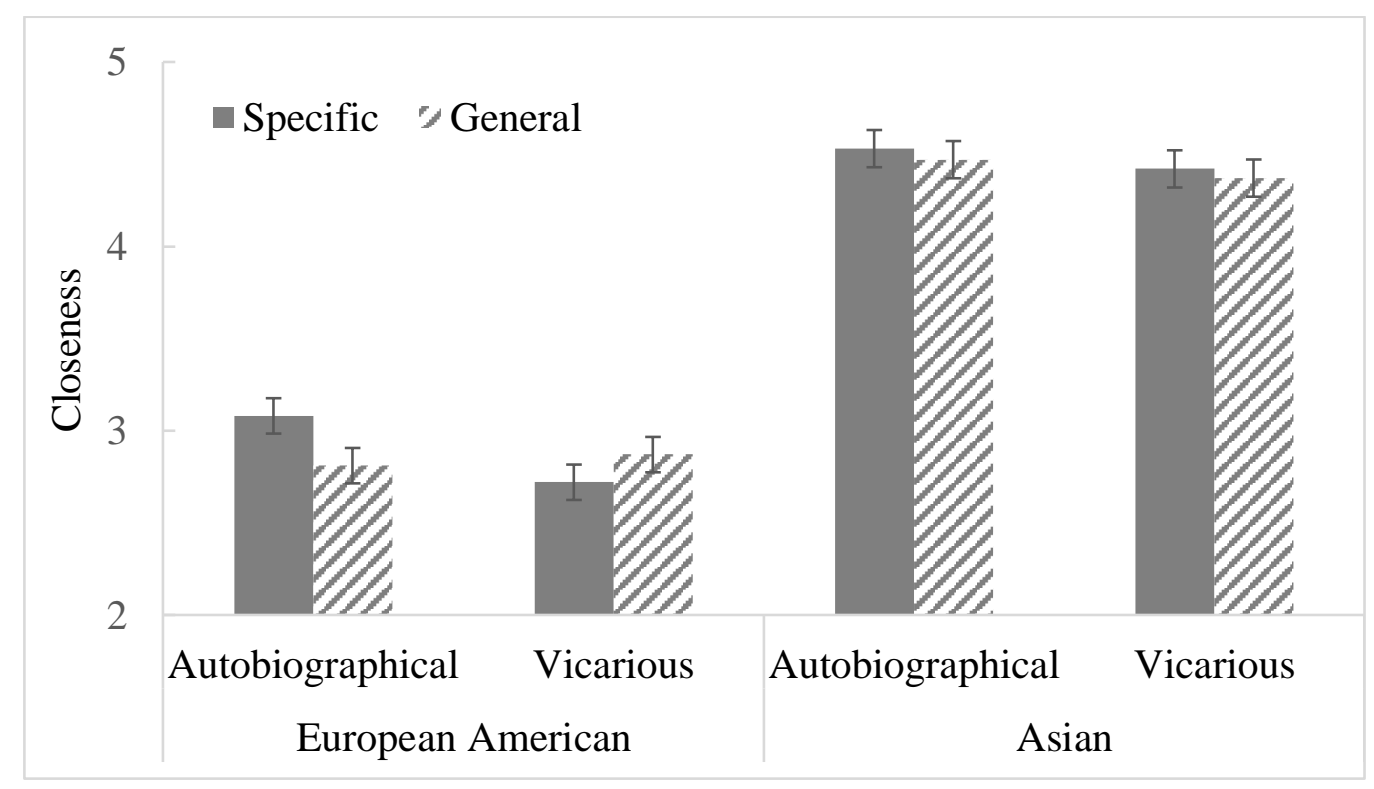

Figure 1. Adjusted mean closeness ratings as a function of the type of memory sharing and culture. Error bars represent standard errors of the adjusted means.

Interdependent value orientation and perceived closeness. A composite score of self-construal was calculated by subtracting independence score from interdependence score for each participant to index the degree of an interdependent value orientation. Consistent with previous studies (e.g., Holland et al., 2004; Wang, 2001), Asians $(M=.41, S D=8.52)$ scored higher on interdependent value orientation 
than did European Americans $(M=-7.93, S D=19.29), F(1,404)=10.59, p=.001, \eta_{\mathrm{p}}{ }^{2}$ $=.026$, independent of age, gender, and education .

Linear regression analyses were conducted to test the relation of participants' interdependent value orientation to their perceived closeness following memory sharing, with culture, age, gender, and education included in the models. The patterns of results were consistent across different types of memory sharing. We thus report results of the mean closeness score across the four memory types as the outcome variable. The regression model showed that, independent of culture, age, gender, and education, an interdependent value orientation was positively associated with perceived closeness following memory sharing, $\beta=.13, t(404)=3.10, p=.002$. The more interdependently oriented a participant was, the closer he or she felt about an imagined conversation partner after sharing memories. In contrast, a linear regression on perceived closeness following conversations about non-person information showed no significant effect of value orientation, $\beta=.07, t(404)=1.66, p=.097$. Additional analyses were conducted with Culture $\mathrm{x}$ Value orientation interaction included in the above models. The interaction was nonsignificant and the pattern of results remained identical.

Perceived closeness and well-being. Asians $(M=45.14, S D=6.91)$ and European Americans $(M=42.76, S D=9.04)$ scored similarly on the Flourishing Scale, $F(1,404)=$ $3.18, p=.075, \eta_{\mathrm{p}}^{2}=.008$, independent of age, gender, and education. Linear regression analyses were conducted to test the relation between participants' perceived closeness following memory sharing in hypothetical situations and their psychological well-being as indexed by the flourishing score, with culture, age, gender, and education included in the models. The patterns of results were consistent across different types of memory sharing and we therefore report results pertaining to the mean closeness score across the four memory 
types as the predictor. Independent of culture, age, gender, and education, perceived closeness was a positive predictor for well-being, $\beta=.25, t(404)=4.48, p<.001$. The closer a participant felt towards a conversation partner following imagined memory sharing, the greater well-being he or she exhibited. A linear regression with perceived closeness following conversations about non-person information as the predictor for well-being showed a similar but weaker effect, $\beta=.18, t(404)=3.12, p=.002$. Additional analyses were conducted with Culture $x$ Perceived closeness interaction included in the above models. The interaction was nonsignificant and the pattern of results remained identical.

\section{Study 2}

Given the original method that we developed to study the social functions of memory sharing, there were some limitations pertaining to the task. Across all scenarios, participants were asked to imagine being the listener of hypothetical conversations. It is unclear whether being the listener versus being the sharer would play a role in subsequent perceived relationship closeness. In addition, some of the scenarios (e.g., about roommate) were not relevant to older participants and the pair of scenarios in some categories (e.g., general vicarious memory) were not as similar as planned. To address these issues, we replicated in Study 2 the main findings of Study 1 on perceived relationship closeness following hypothetical memory conversations.

\section{Method}

Participants. A total of 304 participants were recruited through Amazon Mechanical Turk, including 155 self-identified as White or European American (91 males and 64 females; all except 21 were located in the US) and 149 self-identified as Asian or Asian American (96 males and 53 females; 80\% were located outside the US). We also collected separate identifiers for Asians $(n=92)$ and Asian Americans $(n=57)$. Preliminary analyses showed no systematic differences between the two subgroups and they were combined into 
one “Asian” group. Participants' ages ranged from 18 to 69 years $(M=34.24, S D=9.82)$.

Among the participants, 19 received high school education or less, 76 had college degrees, and 209 had graduate degrees. An additional 41 participants did not complete the survey or follow the instructions and another 67 participants self-identified as belonging to other ethnic groups; those participants were excluded from the study.

Procedure and measures. The hypothetical conversation task was the same as the one used in Study 1 except the following changes. For the pair of scenarios in each type of information sharing, participants were asked to imagine being the sharer in one scenario and being the listener in the other scenario. The "sharer" and "listener" scenarios were counterbalanced such as half of the participants in each cultural group were the sharer in one scenario and the other half of the participants were the sharer in the other scenario. The content of each pair of scenarios was closely matched and was appropriate for participants of all ages (see Table 3). The length of the scenarios was similar across the different types of information sharing; it ranged from 55 to 62 words $(M=58.7, S D=2.6){ }^{2}$

Table 3

Conversation Scenario Excerpts by Information Sharing Type

Information Scenario excerpts

sharing type

Specific "My last birthday was so much fun... I had a blast going to my favorite autobiographical restaurant..."

memory

"I went with my family to the Point Dume State Beach last Saturday. I had so much fun snorkelling off the beach..."

General "I love to be outdoors... My family and I used to go there every spring autobiographical and summer..."

memory

"I like to sing. I used to be in an Acapella club...I enjoyed singing with my clubmates..."

\footnotetext{
${ }^{2}$ Participants also recalled memories of recent conversations. The data addressed separate research questions and were not included in this paper.
} 
Specific

vicarious memory

General vicarious memory

Non-person information
"My friend Ada visited her cousin a month ago... Her cousin took her to Broadway for the musical Lion King..."

"My nephew had his first date with his girlfriend last weekend...He took her to a fancy restaurant for dinner..."

"My cousin Rachel likes to go to gym... She went to gym with her workout buddies every weekday..."

"My friend Victor is good at language learning... Every summer he went to Germany with his classmates to travel..."

"New York City has a humid subtropical climate..."

"Yellowstone National Park is a large wilderness recreation area..."

Following each conversation description, participants rated their feelings of closeness to the imagined conversation partner in four questions, same as in Study 1. Cronbach's alphas for the ratings on the four questions ranged from .92 to .97 across the 10 conversations. The four ratings were thus averaged for each conversation to index the degree of perceived closeness following the conversation.

\section{Results}

Preliminary analyses showed that European Americans $\left(M_{\text {age }}=35.77, S D=11.49\right)$ were on average older than Asians $\left(M_{\mathrm{age}}=32.64, S D=7.42\right), t(265)=2.84, p=.005, d=.32$, and Asians had higher educational levels than European Americans, $\chi^{2}(2, \mathrm{~N}=304)=11.36$, $p=.003, \varphi=.19$. Analyses with and without the demographic variables as covariates yielded identical patterns of results. We report the results of analyses with the covariates.

As in Study 1, we examined the participants' perceived closeness with the imagined conversation partner as the function of the type of information sharing and culture. Across the 10 hypothetical conversations, participants' role as the sharer versus listener did not have any significant effect on closeness ratings, $p \mathrm{~s}>.05$, and the ratings did not differ between the two scenarios within each type of information sharing, $p s>.05$. Thus, participants' role in the conversations was not considered further, and 
the ratings were averaged between the two scenarios for each type of information sharing and submitted to analyses. The mean closeness ratings for different types of information sharing are presented in Table 4.

Table 4

Means and Standard Deviations of Perceived Closeness

\begin{tabular}{|c|c|c|c|c|c|c|c|}
\hline & & \multicolumn{2}{|c|}{ All } & \multicolumn{2}{|c|}{$\begin{array}{l}\text { European } \\
\text { Americans }\end{array}$} & \multicolumn{2}{|c|}{ Asians } \\
\hline & & Mean & SD & Mean & $\mathrm{SD}$ & Mean & SD \\
\hline \multirow[t]{2}{*}{ Specific } & Autobiographical & 4.93 & 1.30 & 4.53 & 1.46 & 5.34 & 0.96 \\
\hline & Vicarious & 4.59 & 1.37 & 4.20 & 1.46 & 5.00 & 1.14 \\
\hline \multirow[t]{2}{*}{ General } & Autobiographical & 4.68 & 1.30 & 4.25 & 1.41 & 5.13 & 0.99 \\
\hline & Vicarious & 4.49 & 1.41 & 4.10 & 1.53 & 4.90 & 1.14 \\
\hline Control & Non-person & 4.09 & 1.86 & 3.63 & 1.98 & 4.57 & 1.61 \\
\hline
\end{tabular}

First, to test whether sharing memories would better serve the function of developing relationship closeness than sharing non-person information, a 2(culture: European American versus Asian) x 2(conversational content: memory versus non-person information) repeatedmeasures ANOVA was conducted on the closeness rating, with culture as a between-subjects factor, conversational content as a within-subjects factor, and an error term accounting for within-individual variance. Participants' age, gender, and education were included in the model. The analysis revealed main effects of culture, $F(1,298)=19.00, p<.001, \eta_{\mathrm{p}}{ }^{2}=.060$, and conversational content, $F(1,302)=89.39, p<.001, \eta_{\mathrm{p}}^{2}=.228$. Asians perceived greater closeness than did European Americans regardless of the type of information sharing. Both European Americans, $F(1,154)=49.70, p<.001, \eta_{\mathrm{p}}^{2}=.244$, and Asians, $F(1,148)=39.69$, $p<.001, \eta_{\mathrm{p}}{ }^{2}=.211$, felt closer to the imagined conversation partner after sharing memories than sharing non-person information. There was no interaction between culture and 
conversational content.

Next, we examined the influence of specificity and protagonist of shared memories on perceived closeness. We conducted a 2(culture: European American vs. Asian) x 2(specificity: specific vs. general) x 2(protagonist: autobiographical vs. vicarious) repeatedmeasures ANOVA on the closeness rating, with culture as a between-subjects factor, memory specificity and memory protagonist as within-subjects factors, and an error term of withinindividual variance. Age, gender, and education were included in the model. There were main effects of culture, $F(1,298)=21.59, p<.001, \eta_{\mathrm{p}}^{2}=.068$, specificity, $F(1,302)=39.95, p$ $<.001, \eta_{\mathrm{p}}^{2}=.117$, and protagonist, $F(1,302)=41.31, p<.001, \eta_{\mathrm{p}}{ }^{2}=.120$, qualified by an interaction between specificity and protagonist, $F(1,302)=6.02, p=.015, \eta_{\mathrm{p}}^{2}=.020$. The adjusted mean ratings from the model are shown in Figure 2.

Asians perceived greater closeness than did European Americans across all types of memory sharing. Further repeated-measures ANOVAs within each culture showed that for Asians, conversation scenarios about specific memories led to greater feelings of closeness than those about general memories, $F(1,148)=20.08, p<.001, \eta_{\mathrm{p}}^{2}=.119$, and scenarios about autobiographical memories led to greater feelings of closeness than those about vicarious memories, $F(1,148)=26.71, p<.001, \eta_{\mathrm{p}}^{2}=.153$. In the European American group, there were main effects of specificity, $F(1,154)=20.57, p<.001, \eta_{\mathrm{p}}{ }^{2}=.118$, and protagonist, $F(1,154)=16.20, p<.001, \eta_{\mathrm{p}}^{2}=.095$, as well as a marginally significant Specificity x Protagonist interaction, $F(1,154)=3.35, p=.069, \eta_{\mathrm{p}}^{2}=.021$. For conversations about autobiographical memories, European Americans felt closer to the imagined partner when the memories were specific than general, $F(1,154)=14.96, p<.001, \eta_{\mathrm{p}}{ }^{2}=.089$, whereas this difference was not significant for vicarious memories, $F(1,154)=3.41, p$ $=.082, \eta_{\mathrm{p}}^{2}=.020$. Overall, for European Americans, sharing specific autobiographical memories with an imagined partner led to greater feelings of closeness than sharing other 
types of memories.

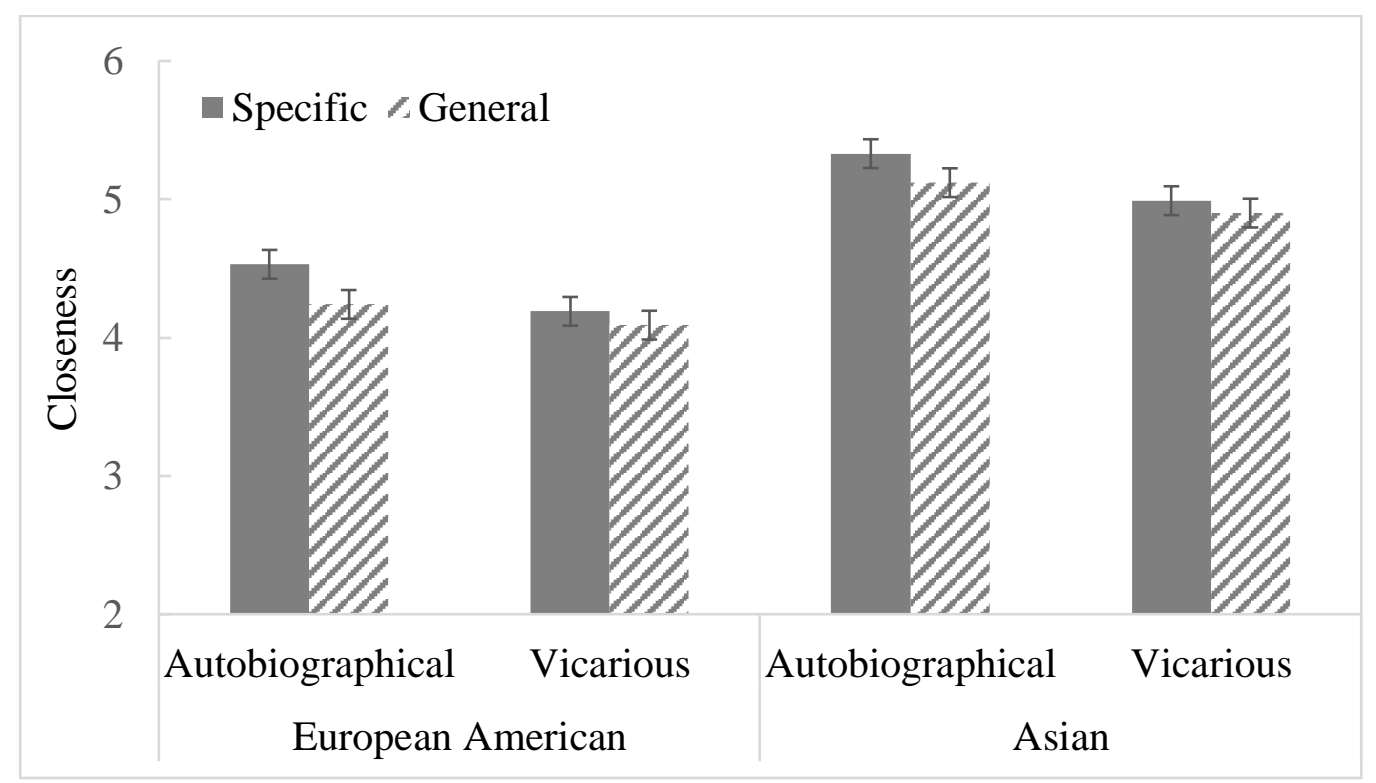

Figure 2. Adjusted mean closeness ratings as a function of the type of memory sharing and culture. Error bars represent standard errors of the adjusted means.

\section{General Discussion}

Research to date has focused on the social functions of specific autobiographical memories in Western populations. Using hypothetical scenarios, the present research is the first to examine in a cross-cultural context the effect of sharing different types of memories on developing relationship closeness and the relation of perceived closeness to psychological well-being. We developed and replicated a novel method in which participants reported their feelings of closeness to a conversation partner in hypothetical scenarios of information sharing. The two studies revealed generally consistent patterns of results. For both European Americans and Asians, sharing memories led to greater feelings of closeness with the imagined partner than sharing non-person information. This is consistent with the notion that memory and memory sharing serve social functions of relationship development and 
maintenance (e.g., Alea \& Bluck, 2007; Bluck, 2003; Pillemer, 2001).

Furthermore, in both studies, Asians perceived greater relationship closeness than did European Americans across all conversational contexts. Given their interdependent value orientation and social sensitivity (Markus \& Kitayama, 1991; Setlock et al., 2004; Wang, 2013), Asians may be particularly attuned to interpersonal exchanges that involve any kind of communication and expect relationship development as a result of the exchanges. In contrast, European Americans tend to view themselves as independent and separate from others and may therefore be less sensitive to social exchanges to experience relationship closeness. This finding was further confirmed at the individual level in Study 1, whereby, independent of culture, participants with a greater interdependent value orientation perceived greater relationship closeness following imagined memory sharing of all types.

Also as predicted, memory protagonist and memory specificity influenced perceived relationship closeness with the imagined conversation partner. For European Americans in both studies, sharing specific autobiographical memories had the strongest impact on perceived relationship closeness. This is in line with extant research showing that memories for distinct, momentary past events happening to oneself play an important role for social bonding particularly among Western individuals (Kulkofsky et al., 2009, 2010; Maki et al., 2015). Such memories are often rich in sensory details and subjective experiences, which help to engage listeners in a joint mental time travel and elicit empathic responses during the conversation, thus facilitating intimacy (Alea \& Bluck, 2003; Baron \& Bluck, 2009; Pillemer, 2001; Pillemer et al., 2015; Rasmussen \& Habermas, 2011).

Similarly, in both studies, Asians also felt closer to the imaged partner when the conversation involved autobiographical than vicarious memories, consistent with the notion that autobiographical memories are more effective for facilitating interpersonal connections than vicarious memories (Cohen, 1998; Rasmussen \& Habermas, 2011; Pillemer et al., 2015). 
The effect of memory specificity on perceived closeness was less stable among Asians and only emerged in Study 2. Wang (2013) contends that general memories often concern rules and regularities that inform individuals of social conventions and thus facilitate interpersonal harmony and group cohesion. Sharing general memories may therefore serve important relationship or group functions in the Asian cultural context. Importantly, culture may further interact with the context of memory usage (private recall vs. social sharing) and the nature of relationships in determining the effect of memory specificity on relationship closeness (Beike et al, 2017; Kulkofsky et al., 2010; Waters et al., 2014).

Finally, independent of culture and other demographic factors, participants who perceived greater relationship closeness following imagined conversations - memory conversations in particular - scored higher on psychological well-being. This is consistent with previous findings that relationship quality, perceived or actual, is a universal key factor in predicting well-being (Baumeister \& Leary, 1995; Ryan \& Deci, 2000; Umberson et al., 2010; Warren et al., 2000). The finding further highlights memory sharing as an important context for relationship closeness and individual psychological functioning.

Some limitations of the current research warrant acknowledgement. Our participants only imagined having conversations. Although studying memory and other cognitive processes with hypothetical scenarios is an informative approach both within and across cultures (e.g., Goyal et al., 2020; Wang \& Ross, 2005; Zhao \& Kushnir, 2019), additional research using other methods are called for to fully understand the correlates and outcomes of memory sharing. In particular, methods involving natural conversations coupled with experimental controls are much needed. Also, although the current research included samples with a more diverse and representative demographic profile than in-lab studies with college students, some of the effects were small and the findings need to be corroborated in more controlled settings. Furthermore, this research focused on new relationships of equal 
partnership, which might explain why the role of listener versus sharer did not affect perceived closeness with the partner. Future research should examine other types of relationship dynamics in affecting memory sharing and intimacy across cultures. Finally, specific and general memories differ in many dimensions (e.g., mental time travel, sensory and emotional details, personal meaning making) (Pillemer, 2001; Wang, 2013). Future research should tackle these dimensions separately to understand the nuances of influences of memory specificity.

In conclusion, the present findings provide additional insights into the social functions of memory and memory sharing. Both memory characteristics and the participants' cultural backgrounds influence feelings of closeness following imagined memory conversations. The findings further suggest that sharing memories not only brings people closer but is also associated with psychological well-being, which has important application values in real life. 


\section{Author Contributions}

Both authors were involved in the research design of the study. Q.W. provided conceptual and methodological guidance. L.G. contributed to data collection and performed data analysis. Both authors worked on the manuscript and approved the final version for submission. 


\section{References}

Alea, N., \& Bluck, S. (2003). Why are you telling me that? A conceptual model of the social function of autobiographical memory. Memory, 11, 165-178.

Alea, N., \& Bluck, S. (2007). I'll keep you in mind: The intimacy function of autobiographical memory. Applied Cognitive Psychology, 21, 1091-1111. https://doi.org/10.1002/acp.1316

Alea, N., \& Wang, Q. (2015). Going global: The functions of autobiographical memory in cultural context. Memory, 23, 1, 1-10. doi:10.1080/09658211.2014.972416

Aron, A., Aron, E. N., \& Smollan, D. (1992). Inclusion of other in the self scale and the structure of interpersonal closeness. Journal of Personality and Social Psychology, 63(4), 596.

Baron, J. M., \& Bluck, S. (2009). Autobiographical memory sharing in everyday life: Characteristics of a good story. International Journal of Behavioral Development, 33(2), 105-117.

Baumeister, R. F., \& Leary, M. R. (1995). The need to belong: desire for interpersonal attachments as a fundamental human motivation. Psychological bulletin, 117(3), 497.

Beike, D. R., Brandon, N. R., \& Cole, H. E. (2016). Is sharing specific autobiographical memories a distinct form of self-disclosure?. Journal of Experimental Psychology: General, 145(4), 434.

Beike, D. R., Cole, H. E., \& Merrick, C. R. (2017). Sharing specific “we” autobiographical memories in close relationships: The role of contact frequency. Memory, 25(10), 14251434.

Bluck, S. (2003). Autobiographical memory: Exploring its functions in everyday life. Memory, 11(2), 113-123. 
Bluck, S., Alea, N., Habermas, T., \& Rubin, D. C. (2005). A tale of three functions: The selfreported uses of autobiographical memory. Social cognition, 23(1), 91.

Brewer, W.F. (1986). What is autobiographical memory? In D.C. Rubin (Ed.), Autobiographical memory (pp. 25-49). Cambridge: Cambridge University Press.

Brint, S. (2001). Gemeinschaft revisited: A critique and reconstruction of the community concept. Sociological theory, 19(1), 1-23.

Bruce, D. (1989). Functional explanations of memory. In L. W. Poon, D. C. Rubin, \& B. A. Wilson (Eds.), Everyday cognition in adulthood and late life (pp. 44-58). Cambridge: Cambridge University Press.

Chen, G. M. (1995). Differences in self-disclosure patterns among Americans versus Chinese: A comparative study. Journal of Cross-Cultural Psychology, 26(1), 84-91.

Cialdini, R. B., Brown, S. L., Lewis, B. P., Luce, C., \& Neuberg, S. L. (1997). Reinterpreting the empathy-altruism relationship: When one into one equals oneness. Journal of personality and social psychology, 73(3), 481.

Cohen, G. (1998). The effects of aging on autobiographical memory. Autobiographical memory: Theoretical and applied perspectives, 105-123.

Conway, M. A., \& Pleydell-Pearce, C. W. (2000). The construction of autobiographical memories in the self-memory system. Psychological Review, 107 (2), 261-288. doi:10.1037/0033-295x.107.2.261.

Cross, S. E., \& Morris, M. L. (2003). Getting to know you: The relational self-construal, relational cognition, and well-being. Personality and Social Psychology Bulletin, 29(4), $512-523$

Demiray, B., Mischler, M., \& Martin, M. (2019). Reminiscence in everyday conversations: A naturalistic observation study of older adults. The Journals of Gerontology: Series B, 74(5), 745-755. 
Diener, E., Wirtz, D., Tov, W., Kim-Prieto, C., Choi, D., Oishi, S., \& Biswas-Diener, R. (2009). New measures of well-being: Flourishing and positive and negative feelings. Social Indicators Research, 39, 247-266.

Faul, F., Erdfelder, E., Lang, A.-G., \& Buchner, A. (2007). G*Power 3: A flexible statistical power analysis program for the social, behavioral, and biomedical sciences. Behavior Research Methods, 39(2), 175-191.

Gächter, S., Starmer, C., \& Tufano, F. (2015). Measuring the closeness of relationships: a comprehensive evaluation of the'Inclusion of the Other in the Self'scale. PloS one, 10(6).

Goyal, N., Adams, M., Cyr, T. G., Maass, A., \& Miller, J. G. (2020). Norm-based spontaneous categorization: Cultural norms shape meaning and memory. Journal of Personality and Social Psychology, 118(3), 436-56. doi:10.1037/pspi0000188.

Holland, R. W., Roeder, U. R., Rick B. van, B., Brandt, A. C., \& Hannover, B. (2004). Don't stand so close to me: The effects of self-construal on interpersonal closeness. Psychological science, 15(4), 237-242.

Kito, M. (2005). Self-disclosure in romantic relationships and friendships among American and Japanese college students. The Journal of Social Psychology, 145, 127-140.

Kito, M., Yuki, M., \& Thomson, R. (2017). Relational mobility and close relationships: A socioecological approach to explain cross-cultural differences. Personal Relationships, 24(1), 114-130.

Kulkofsky, S., Wang, Q., \& Hou, Y. (2010). Why I remember that: The influence of contextual factors on beliefs about everyday memory. Memory \& Cognition, 38, 461473.

Kulkofsky, S., Wang, Q., \& Koh, J. B. K. (2009). Functions of memory sharing and motherchild reminiscing behaviors: Individual and cultural variations. Journal of Cognition and Development, 10, 92-114. doi: 10.1080/15248370903041231 
Maki, Y., Kawasaki, Y., Demiray, B., \& Janssen, S. M. (2015). Autobiographical memory functions in young Japanese men and women. Memory, 23(1), 11-24.

Markus, H. R., \& Kitayama, S. (1991). Culture and the self: Implications for cognition, emotion, and motivation. Psychological review, 98(2), 224.

Miller, P. J. (2009). Stories have histories: Reflections on the personal in personal storytelling. Taiwan Journal of Anthropology, 7, 67- 84.

Neff, K. D., Pisitsungkagarn, K., \& Hsieh, Y. P. (2008). Self-compassion and self-construal in the United States, Thailand, and Taiwan. Journal of Cross-Cultural Psychology, 39(3), 267-285.

Nelson, K., \& Fivush, R. (2004). The emergence of autobiographical memory: a social cultural developmental theory. Psychological review, 111(2), 486.

Oppenheim, D. (2006). Child, parent, and parent-child emtoion narratives: Implications for developmental psychopathology. Development and Psychopathology, 18, 771-790. doi: 10.1017/S095457940606038X

Pasupathi, M., \& Carstensen, L. L. (2003). Age and emotional experience during mutual reminiscing. Psychology and aging, 18(3), 430.

Pillemer, D. B. (2001). Momentous events and the life story. Review of general psychology, 5(2), 123-134.

Pillemer, D. B., Steiner, K. L., Kuwabara, K. J., Thomsen, D. K., \& Svob, C. (2015). Vicarious memories. Consciousness and Cognition, 36, 233-245.

Rasmussen, A. S., \& Habermas, T. (2011). Factor structure of overall autobiographical memory usage: The directive, self and social functions revisited. Memory, 19(6), 597605.

Reese, E., Fivush, R., Merrill, N., Wang, Q., \& McAnally, H. (2017). Adolescents' intergenerational narratives across cultures. Developmental Psychology, 53, 6, 1142 - 
1153. http://dx.doi.org/10.1037/dev0000309

Repetti, R. L., Taylor, S. E., \& Seeman, T. E. (2002). Risky families: family social environments and the mental and physical health of offspring. Psychological bulletin, $128(2), 330$.

Rubin, D. C. (2005). A basic-systems approach to autobiographical memory. Current Directions in Psychological Science, 14(2), 79-83.

Ryan, R. M. \& Deci, E. L. (2000). Self-determination theory and the facilitation of intrinsic motivation, social development, and well-being. American Psychologist, 55, 68-78.

Schug, J., Yuki, M., \& Maddux, W. (2010). Relational Mobility Explains Between- and Within-Culture Differences in Self-Disclosure to Close Friends. Psychological Science, 21(10), 1471-1478. doi: 10.1177/0956797610382786

Setlock, L D., Fussell, S. R., \& Neuwirth. C. (2004). Taking it out of context: collaborating within and across cultures in face-to-face settings and via instant messaging. Proceedings of CSCW 2004 (pp. 604-613), NY: ACM Press.

Singelis, T. M. (1994). The Measurement of Independent and Interdependent Self-Construals. Personality and Social Psychology Bulletin, 20(5), 580-591.

Song, Q., Koh, J. B. K., \& Wang, Q. (2018). Children's narrative representations of peer experiences in cultural contexts: The relations to psychological adjustment. Journal of Child and Family Studies, 27(6), 2037-2048. doi:10.1007/s10826-018-1033-4

Sparks, S., Cunningham, S. J., \& Kritikos, A. (2016). Culture modulates implicit ownershipinduced self-bias in memory. Cognition, 153, 89-98. doi:10.1016/j.cognition.2016.05.003

Thomsen, D. K., \& Pillemer, D. B. (2017). I know my story and I know your story: Developing a conceptual framework for vicarious life stories. Journal of Personality, 85(4), 464-480. 
Townsend, M. A., McCracken, H. E., \& Wilton, K. M. (1988). Popularity and intimacy as determinants of psychological well-being in adolescent friendships. The Journal of Early Adolescence, 8(4), 421-436.

Tulving, E. (2002). Episodic memory: From mind to brain. Annual Review of Psychology, 53: $1-25$.

Umberson, D., Crosnoe, R., \& Reczek, C. (2010). Social relationships and health behavior across the life course. Annual review of sociology, 36, 139-157.

Wang, H-C., Fussell, S. R. \& Setlock, L. D. (2009). Cultural difference and adaptation of communication styles in computer-mediated group brainstorming. Proc. CHI 2009 (pp. 669-678). NY: ACM.

Wang, Q. (2001). Cultural effects on adults' earliest childhood recollection and selfdescription: Implications for the relation between memory and the self. Journal of Personality and Social Psychology, 81, 220-233.

Wang, Q. (2004). The cultural context of parent-child reminiscing: A functional analysis. In M. W. Pratt \& B. Fiese (Eds.), Family stories and the life course: Across time and generations (pp. 279-301). Mahwah, NJ: Lawrence Erlbaum Associates.

Wang, Q. (2006). Earliest recollections of self and others in European American and Taiwanese young adults. Psychological Science, 17, 708-714.

Wang, Q. (2013). The autobiographical self in time and culture. Oxford University Press.

Wang, Q. (2018). Culture in collaborative remembering. In M.L. Meade, C.B. Harris, P. Van Bergen, J. Sutton, \& A.J. Barnier (Eds.), Collaborative Remembering: Theories, Research, and Applications. Oxford University Press.

Wang, Q., \& Conway, M. A. (2004). The stories we keep: Autobiographical memory in American and Chinese middle-aged adults. Journal of personality, 72(5), 911-938. 
Wang, Q. \& Leichtman, M. D. (2000). Same beginnings, different stories: A comparison of American and Chinese children's narratives. Child Development, 71, 5, 1329-1346.

Wang, Q., \& Ross, M. (2005). What we remember and what we tell: The effects of culture and self-priming on memory representations and narratives. Memory, 13(6), 594-606.

Wang, Q., \& Ross, M. (2007). Culture and memory. In S. Kitayama \& D. Cohen (Eds.), Handbook of cultural psychology (pp. 645-667). New York: Guilford Press.

Warren, S. L., Emde, R. N., \& Sroufe, L. A. (2000). Internal representations: Predicting anxiety from children's play narratives. Journal of the American Academy of Child and Adolescent Psychiatry, 39, 100-107. doi:10.1097/00004583-200001000-00022

Waters, T. E., Bauer, P. J., \& Fivush, R. (2014). Autobiographical memory functions served by multiple event types. Applied Cognitive Psychology, 28(2), 185-195.

Williams, J. M., \& Broadbent, K. (1986). Autobiographical memory in suicide attempters. Journal of abnormal psychology, 95(2), 144-149. doi: 10.1037/0021$843 X .95 .2 .144$

Yoon, C., Hasher, L., Feinberg, F., Rahhal, T. A., \& Winocur, G. (2000). Cross-cultural differences in memory: The role of culture-based stereotypes about aging. Psychology and Aging, 15(4), 694.

Yuki, M., \& Schug, J. (2012). Relational mobility: A socioecological approach to personal relationships. In O. Gillath, G. E. Adams, \& A. D. Kunkel (Eds.), Relationship science: Integrating evolutionary, neuroscience, and sociocultural approaches (pp. 137-151). American Psychological Association.

Yuki, M., Schug, J., Horikawa, H., Takemura, K., Sato, K., Yokota, K., \& Kamaya, K. (2007). Development of a scale to measure perceptions of relational mobility in society (CERSS Working Paper 75). Sapporo, Japan: Center for Experimental Research in Social Sciences, Hokkaido University. 
Zahn-Waxler, C., Park, J., Usher, B., Belouad, F., Cole, P., \& Gruber, R. (2008). Young children's representations of conflict and distress: A longitudinal study of boys and girls with disruptive behavior problems. Development and Psychopathology, 20, 99-119. http://dx.doi.org/10.1017/S0954579408000059

Zhao, X., \& Kushnir, T. (2019). How U.S. and Chinese Children Talk about Personal, Moral and Conventional Choices. Cognitive Development, 52. doi:10.1016/j.cogdev.2019.100804 